\title{
Adaptive Delay Tolerant Routing Protocol (ADTRP) for Cognitive Radio Mobile Ad Hoc Networks
}

\author{
L. Indhumathi \\ M.Phil Research Scholar \\ Department of Information Technology \\ Bharathiar University, Coimbatore, India
}

\author{
R. Vadivel, PhD \\ Assistant Professor \\ Department of Information Technology \\ Bharathiar University, Coimbatore, India
}

\begin{abstract}
A cognitive radio network (CRN) is a kind of wireless network that consists of wireless devices embedded with cognitive radios that can sense the available channels in the neighborhood and switch the communication channel, if needed. Delay tolerant routing is a challenging task in such network. Hence this paper proposes an adaptive delay tolerant routing protocol shortly termed as ADTRP for cognitive radio mobile ad hoc networks. The performance metrics such as throughput, packet delivery ratio and delay are chosen. Simulations are carried out using cognitive radio cognitive network (CRCN) simulator and the results shows that the proposed protocol ADTRP performs better in terms of improved throughput, better packet delivery ratio, decreased packet drop and reduced delay.
\end{abstract}

\section{Keywords}

Cognitive radio, delay, routing, channel, throughput.

\section{INTRODUCTION}

A cognitive radio mobile ad hoc network (CRMANET) denotes to a MANET formed by mobile nodes with cognitive radios. It consists of mobile primary users (PUs) and mobile secondary users (SUs) [14]. Also there are a primary channel and also a secondary channel which is substandard to the primary one in terms of bandwidth, channel quality, etc. The PUs communicate over the primary channel, while the SUs mainly use the secondary channel and can sometimes toggle to the primary channel only in the regions free of PU communications. An consummate CRMANET of this kind is one deployed for military actions or post-disaster rescue, where the Pus and SUs correspond to some remarkable nodes coordinating the network mission(e.g., commanding vehicles or personnel)and average mobile nodes, respectively, Enabling cognitive communication in the above scenario can obviously help improve the spectrum efficiency of the primary channel and also the communication performance of the SUs. This research work focused on design and development of adaptive delay tolerant routing protocol for cognitive radio mobile ad-doc networks.

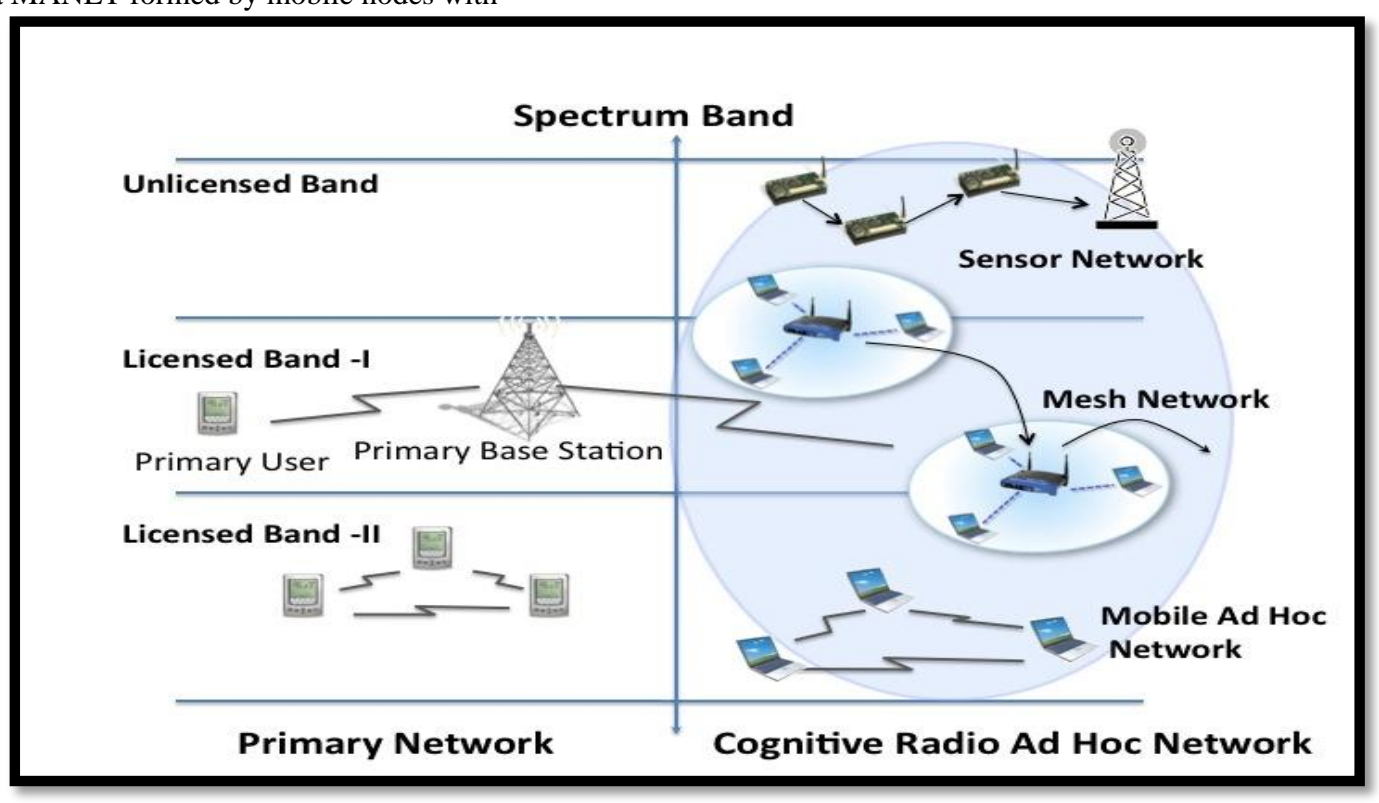

Fig.1. Cognitive Radio Ad hoc Network [y]

\section{LITERATURE REVIEW}

Geographic routing protocols for conventional MANETs motivate the TIGHT design. GFG [15], [16] is a geographic routing algorithm that extracts a connected planar sub graph on which routing is performed. The packet is guaranteed of delivery as long as the unit graph is static and connected during the time to route a message. As the detailed routing protocol of GFG routing algorithm, GPSR [8] supports a greedy mode in which every node, say $\mathrm{X}$, selects the next hop as the neighbor which is closest and also closer than $\mathrm{X}$ to the destination, say D. If a valid next hop exists, the greedy mode continues until the packet reaches D. If no such node exists, the perimeter mode of GPSR is activated to route the packet around the void until the greedy mode can 
resume. TBR [9] is another known geographic routing strategy in which every packet is routed along a physical trajectory to D. GPSR and TBR are nearly stateless, highly scalable, and very insensitive to node mobility. However, since GPSR does not take the PU information into account, directly adopting GPSR to CR-MANETs often leads to longer routing path. Also, how to obtain the optimal trajectory required by TBR in CR-MANETS remains an open challenge. Most of the routing protocols proposed for CR-MANETs so far involve on-demand route discovery and thus require each intermediate node to maintain significant amount of state information. In particular, existing protocols [2]-[4], [6], [7] are adaptations of AODV [13] or DSR [17] to CR-MANETs. They all involve on-demand route discovery to find spectrum aware end-to-end routes as well as route maintenance in case of route breaks due to node mobility or dynamic spectrum availability. SEARCH [5] sends on-demand route requests on every channel using greedy geographic routing, based on which the destination can then derive an optimal, possibly multichannel path which involves multiple anchor nodes with each identifying a unique PU region to be circumvented. As pointed out in [1], SEARCH's path optimization is very sensitive to node mobility and spectrum dynamics. In contrast, our TIGHT protocol is free of route discovery and maintenance, and it inherits the aforementioned merits of GPSR and TBR.

\section{PROPOSED WORK}

The cognitive radio ad hoc network (CRAHN) is a selforganized ad hoc network consists of the licensed PU nodes and the unlicensed SU nodes. Both the SU nodes and PU nodes operate with a limited transmission range and are peers of each other. For simplicity, we assume each PU node to own a distinct licensed channel, identified using the ID of the PU node. An SU node considers a PU channel to be available for use if the corresponding PU node is within the transmission range of the SU node and that the PU node is turned OFF (i.e., not active). There exists an SU-SU link if the two end SU nodes are within the transmission range of each other and there exists at least one common available PU channel in the mutually intersecting neighborhood of the two SU nodes. Depending on the activity status of the PU nodes, the set of PU channels in the neighborhood of the SU nodes changes dynamically with time. Thus, even in a static network of SU nodes and PU nodes, communication topologies (like paths and trees) that connect the SU nodes may have to be frequently reconfigured depending on the availability of the PU channels in the SU-SU neighborhoods. When the propagation delay $\tau>0$, it takes at least time $\tau$ for the message to traverse a distance, which imposes a lower bound on the ratio of the minimum multihop delay (MMD) to the source - destination distance. This implies that the MMD scales at least linearly with the source-destination distance.

The positive propagation delay $\tau$ also imposes an upper bound $\mathrm{TS} / \tau$ on the maximum number of hops that the message can traverse in a primary slot TS. For an instantaneously connected network, this upper bound can be actually attained in the infinite connected component consisting of communication links. But for an intermittently connected network, this upper bound may probably not be attained due to the limited diameter of the finite connected components formed by communication links, especially when the propagation delay $\tau$ is small. Specifically, there may not exist a connected component which has a path with $\mathrm{TS} / \tau$ hops. Thus, although the scaling order is always linear, it can be expected that the scaling rate for an instantaneously connected network is much smaller than the one for an intermittently connected network.

Given the complete knowledge of the future availability of the PU channels, the proposed routing algorithm works as follows: At a time instant $t$ for which we want to determine a stable network-wide communication topology (say a shortest path tree rooted at source SU node s), we check if the static $\mathrm{SU}$ graph $\mathrm{G}_{\mathrm{t}}(\mathrm{SU})$ is connected. If all the vertices of the $\mathrm{SU}$ graph can be visited by running the Breadth First Search (BFS) algorithm from any arbitrarily chosen node, then the graph is connected; otherwise, not. We now proceed to the next sampling time instant $\mathrm{t}+1$ and take intersection of graphs $G_{t}(S U)$ and $G_{t+1}(S U)$ and refer to the intersection graph as mobile graph $\mathrm{G}_{\mathrm{t} \cdots \mathrm{t}+1}(\mathrm{SU})=\mathrm{G}_{\mathrm{t}}(\mathrm{SU}) \cap \mathrm{G}_{\mathrm{t}+1}(\mathrm{SU})$. If $\mathrm{G}_{\mathrm{t} \cdots \mathrm{t}+1}(\mathrm{SU})$ is connected, we continue like this until we come across sampling time instants $\mathrm{t}+\mathrm{k}$ and $\mathrm{t}+\mathrm{k}+1$ such that $\mathrm{G}_{\mathrm{t}} \cdots \mathrm{t}+\mathrm{k}$ (SU) is connected and $\mathrm{G}_{\mathrm{t} \cdots t+\mathrm{t}+1}$ (SU) is not connected. If $\mathrm{G}_{\mathrm{t} \cdots \mathrm{t}+\mathrm{k}}(\mathrm{SU})$ is connected, it implies the communication topology of interest exists in each of the static SU graphs $G_{t}$ $(\mathrm{SU}), \mathrm{G}_{\mathrm{t}+1}(\mathrm{SU}), \ldots, \mathrm{G}_{\mathrm{t}+\mathrm{k}}(\mathrm{SU})$. We could use that topology across all time instants $t, t+1, \ldots, t+k$. We repeat the above procedure from time instant $\mathrm{t}+\mathrm{k}+1$ to find the next stable mobile graph and communication topology and continue likewise for the duration of the network session.

The ADTRP algorithm finds a stable sequence of instances of the mobile graph and the communication topology of interest such that the number of transitions from one instance of the topology to another in the sequence is the global minimum. The average lifetime of the mobile graphs in the stable sequence found by the ADTRP algorithm would serve as an upper bound (benchmark) for any communication topology that spans all the SU nodes found by any centralized or distributed algorithm. The ADTRP algorithm is generic and could be used to determine a stable sequence of any communication topology that spans the entire CRAHN network of SU nodes as long as there exists an algorithm or heuristic to determine that communication topology (e.g., shortest path tree, minimum spanning tree, connected dominating set, etc).

The tradeoff we anticipate is that the stable sequence determined for the communication topology of interest may not be optimal with respect to the metric for which the latter is known for. For example, the shortest path tree determined

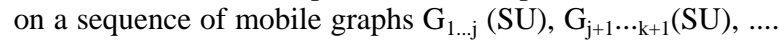
$\mathrm{G}_{\mathrm{r}+1} \ldots \mathrm{T}(\mathrm{SU})$ may not have the same average tree height (a measure of the minimum hop count paths between any two nodes as well as the diameter of the network) compared to the average height of a sequence of shortest path trees determined on the individual static graphs $\mathrm{G}_{1}(\mathrm{SU}), \mathrm{G}_{2}(\mathrm{SU})$, $\ldots, \mathrm{G}_{\mathrm{T}}(\mathrm{SU})$.

This is because, when we aim for stability and determine a mobile graph $G_{t \cdots t+k}(S U)$ that exists for the longest time, the number of SU-SU edges in the mobile graph decreases and we mostly end up with the minimal number of SU-SU edges that would be needed to keep the SU nodes connected. A shortest path tree determined on such a mobile graph $\mathrm{G}_{\mathrm{t}} \cdots \mathrm{t}+\mathrm{k}$ (SU) might have a height larger than the average height of the individual shortest path trees determined on the static graphs $\mathrm{G}_{\mathrm{t}}(\mathrm{SU}), \mathrm{G}_{\mathrm{t}+1}(\mathrm{SU}), \ldots, \mathrm{G}_{\mathrm{t}+\mathrm{k}}(\mathrm{SU})$. 


\section{ADTRP Algorithm}

Begin ADTRP Algorithm

while $(i \leq T)$ do

Find $G_{i \ldots j}(S U)=G_{i}(S U) \cap G_{i+1}(S U) \cap \ldots \cap G=(S U)$

(such that $G_{i \ldots j}(S U)$ is connected and $G_{i \ldots j+l}(S U)$ is not connected)

Stable_G $G_{1 \ldots T}(S U)=$ Stable_G $G_{1 \ldots T}(S U) U\left\{G_{i \ldots j}(S U)\right\}$

Find a shortest path tree $S P T_{i \ldots j}(S U)=B F S\left(G_{i \ldots j}(S U), s\right)$

$A D T R P_{1 \ldots T}(S U)=A D T R P_{1 \ldots T}(S U) U\left\{S P T_{i \ldots j}(S U)\right\}$

$i=j+1$

end while

return $A D T R P_{1 \ldots T}(S U)$ and Stable_G $G_{1 \ldots T}(S U)$

End ADTRP Algorithm

\section{SIMULATION SETTINGS AND PERFORMANCE METRICS}

The simulations are carried our using cognitive radio cognitive network (CRCN) simulator. The cognitive radio cognitive network (CRCN) simulator is a software based network simulator for network-level simulations. It is based on open-source NS-2 (network simulator 2). CRCN simulator supports performance evaluations for the proposed dynamic spectrum resource allocation, power control algorithms, and the adaptive Cognitive Radio (CR) networking protocols including the CR MAC and the CR Routing protocols. This simulator uses NS-2 to generate realistic traffic and topology patterns. For each node in this simulator, a reconfigurable multi-radio multichannel PHY layer is available by customizing the spectrum parameters such as transmission power, propagation and etc. The architecture of $\mathrm{CRCN}$ is shown below

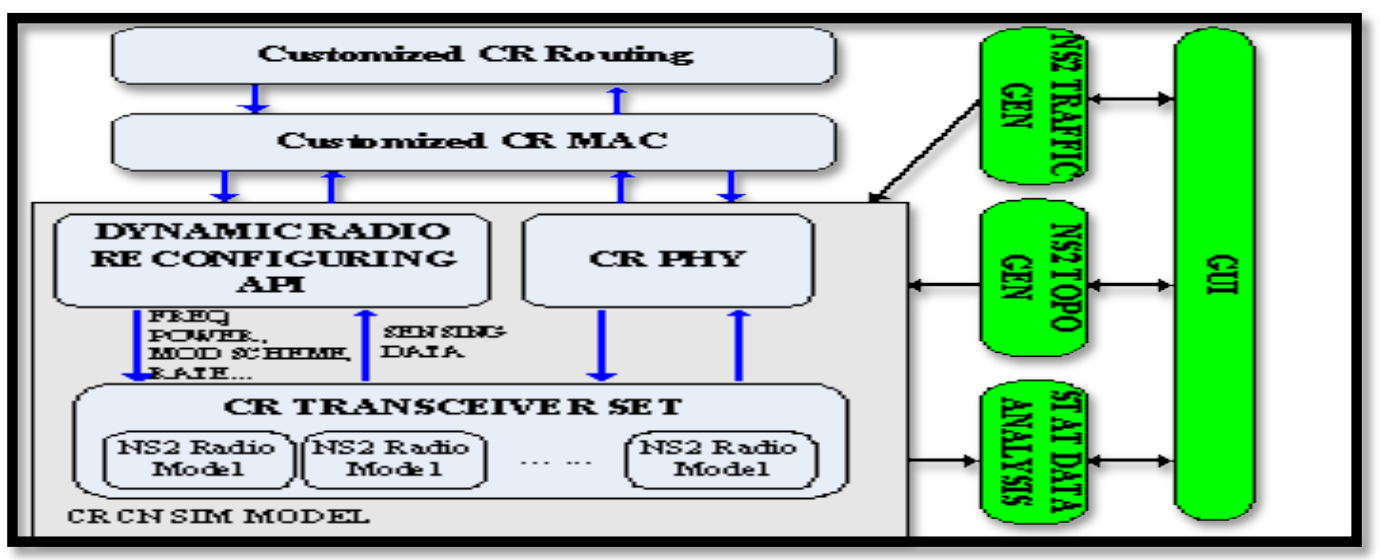

Fig.2. Architecture of CRCN

200 mobile nodes starting from IP address 192.168.1.1 to192.168.1.200 move in a $1500 \times 1000$ meter rectangular region for 100 seconds (simulation time). The channel capacity of mobile nodes is set to 2 Mbos. Distributed Coordination Function (DCF) on IEEE 802.11 is used for wireless LANs. It has the functionality to notify the network layer about link breakage. It is assumed that each node moves independently with the variant mobility speed between 0.5 to $2.5 \mathrm{~m} / \mathrm{s}$. The transmission range has been varied from 150 to 200 meters. The simulated traffic is Constant Bit Rate (CBR). The simulation settings are also represented in tabular format as shown in Table 1.

Table 1. Simulation Settings

\begin{tabular}{|l|l|}
\hline No. of Nodes & 200 \\
\hline Terrain Size & 1500 X $1000 \mathrm{~m}$ \\
\hline MAC & $802.11 \mathrm{~b}$ \\
\hline Radio Transmission Range & $150-200$ meters \\
\hline Simulation Time & 100 seconds \\
\hline Traffic Source & CBR (Constant Bit Rate) \\
\hline Packet Size & 256 Kbits \\
\hline Mobility Model & Random Waypoint Model \\
\hline Speed & $0.5-2.5 \mathrm{~m} / \mathrm{s}$ \\
\hline
\end{tabular}

The following metrics are taken into account for evaluating the proposed routing mechanism with AOMDV, TIGHT and ADTRP protocols.
- Packet Delivery Ratio

- Drop

- Delay

- Throughput 


\section{RESULTS AND DISCUSSIONS}

In Fig. 3 the xgraph result shows the performance analysis of the protocols such as AOMDV, TIGHT and ADTRP in terms of throughput. The values are shown in Table. 2. In Fig.4 the xgraph result shows the performance analysis of the protocols such as AOMDV, TIGHT and ADTRP in terms of packet delivery ratio. The values are shown in Table. 3. In Fig.5 the xgraph result shows the performance analysis of the protocols such as AOMDV, TIGHT and ADTRP in terms of packets drop. The values are shown in Table. 4. In Fig.6 the xgraph result shows the performance analysis of the protocols such as AOMDV, TIGHT and ADTRP in terms of delay. The values are shown in Table. 5.

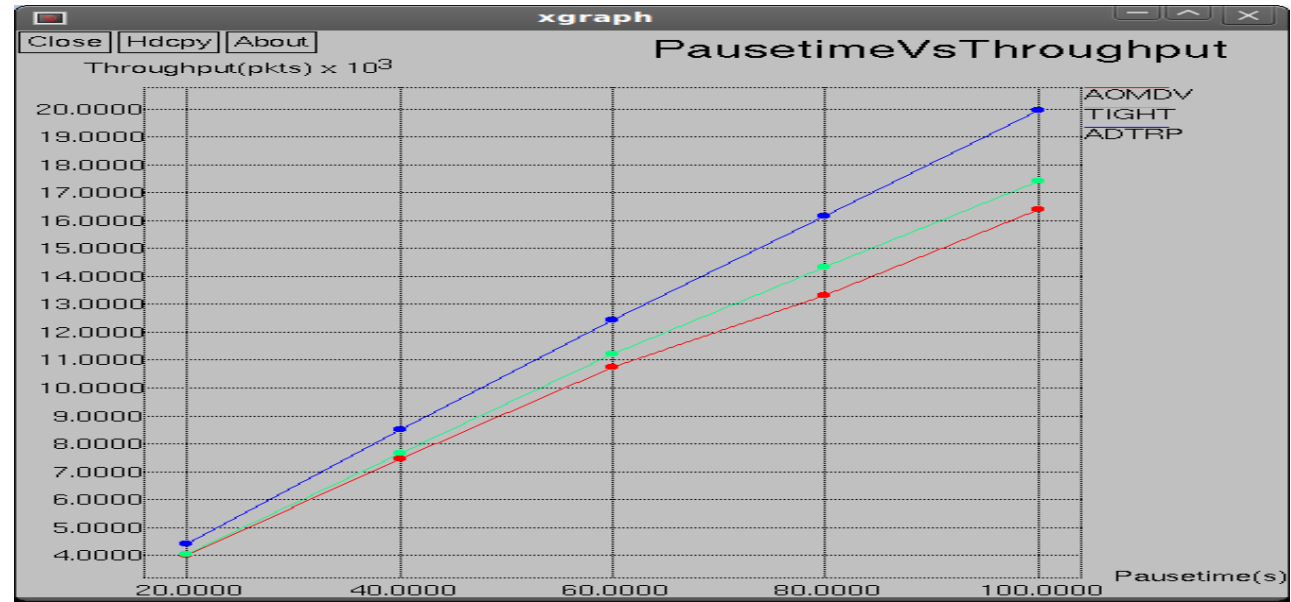

Fig.3. Pause time Vs Throughput

Table.2. Pause time Vs Throughput

\begin{tabular}{|c|c|c|c|}
\hline Nodes & AOMDV & TIGHT & ADTRP \\
\hline 20 & 3994 & 4045 & 4403 \\
\hline 40 & 7475 & 7680 & 8499 \\
\hline 60 & 10752 & 11213 & 12442 \\
\hline 80 & 13312 & 14336 & 16179 \\
\hline 100 & 16384 & 17408 & 19968 \\
\hline
\end{tabular}

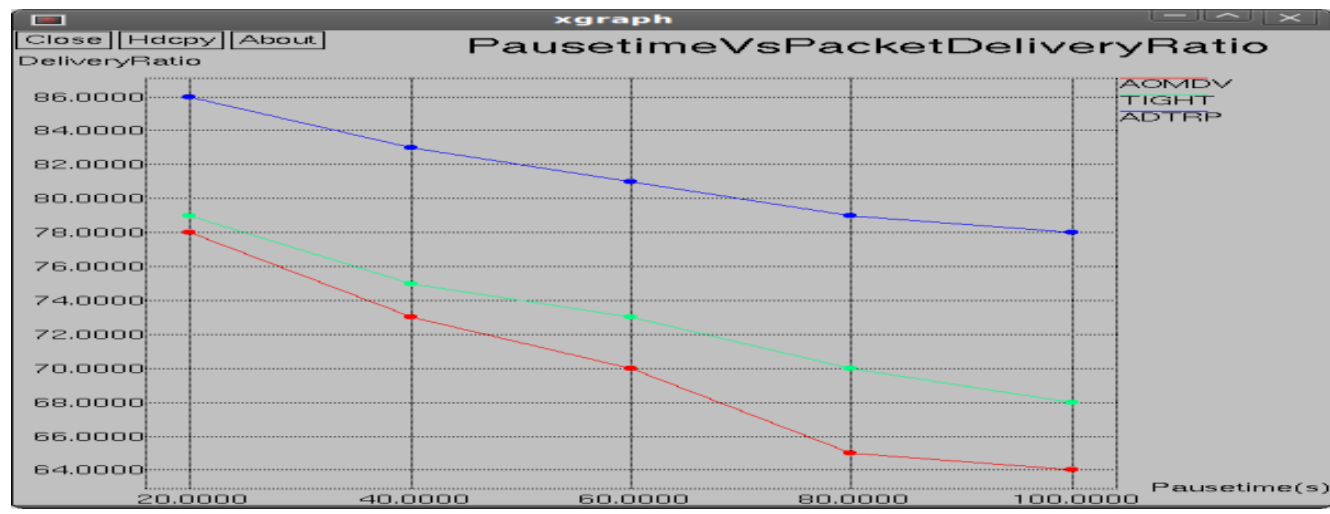

Fig.4. Pause time Vs Packet Delivery Ratio

Table.3. Pause time Vs Packet Delivery Ratio

\begin{tabular}{|c|c|c|c|}
\hline Nodes & AOMDV & TIGHT & ADTRP \\
\hline 20 & 78 & 79 & 86 \\
\hline 40 & 73 & 75 & 83 \\
\hline 60 & 70 & 73 & 81 \\
\hline 80 & 65 & 70 & 79 \\
\hline 100 & 64 & 68 & 78 \\
\hline
\end{tabular}




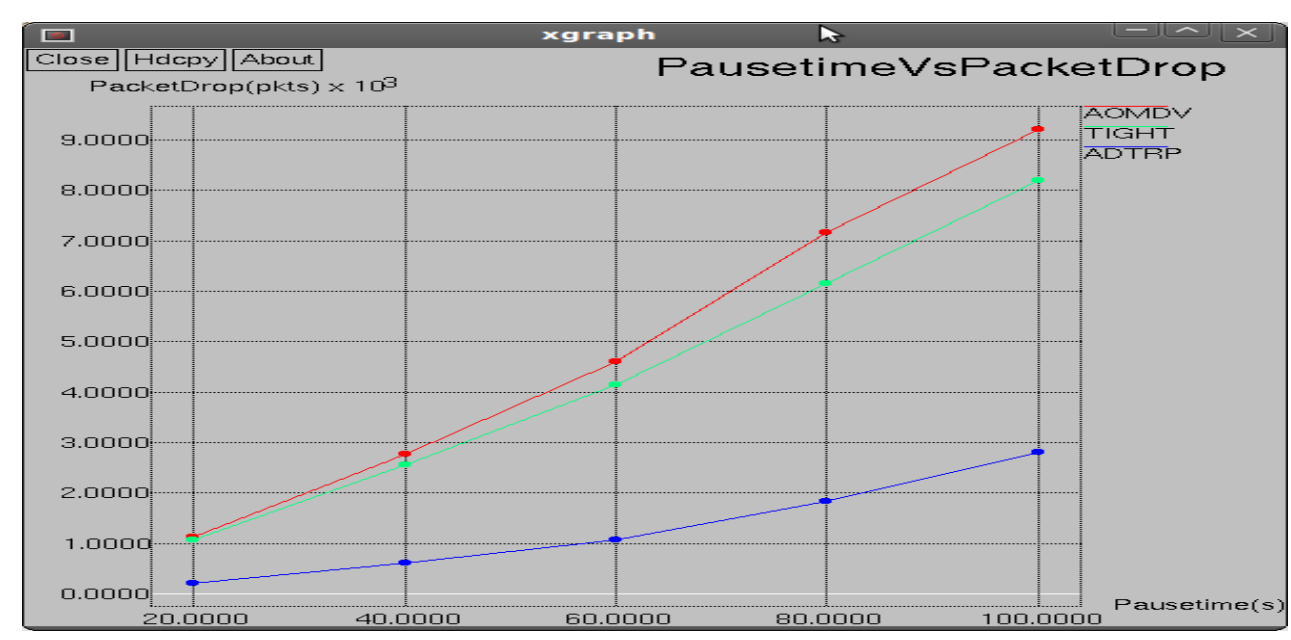

Fig.5. Pause time Vs Packets Drop

Table.4. Pause time Vs Packets Drop

\begin{tabular}{|c|c|c|c|}
\hline Nodes & AOMDV & TIGHT & ADTRP \\
\hline 20 & 1126 & 1075 & 205 \\
\hline 40 & 2765 & 2560 & 614 \\
\hline 60 & 4608 & 4147 & 1075 \\
\hline 80 & 7168 & 6144 & 1843 \\
\hline 100 & 9216 & 8192 & 2816 \\
\hline
\end{tabular}

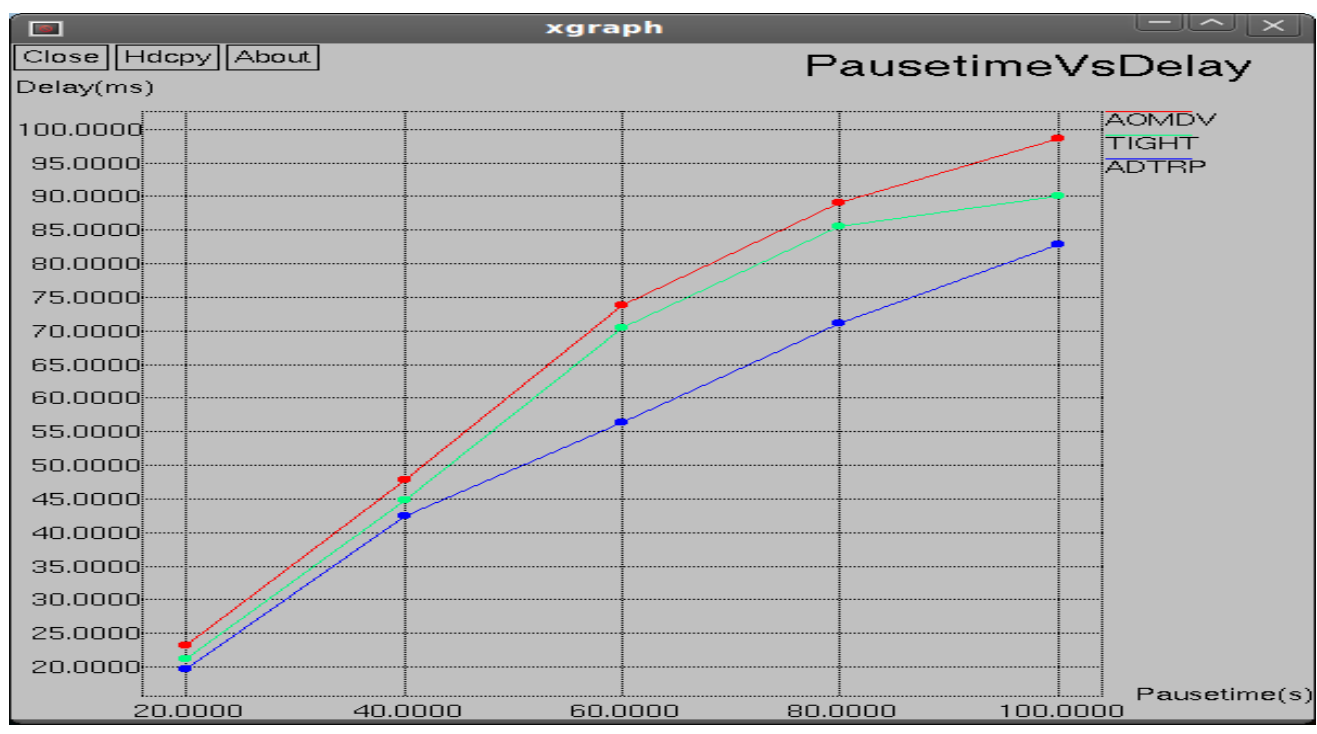

Fig.6. Pause time Vs Delay

Table.5. Pause time Vs Delay

\begin{tabular}{|c|c|c|c|}
\hline Nodes & AOMDV & TIGHT & ADTRP \\
\hline 20 & 23.2 & 21.1 & 19.6 \\
\hline 40 & 47.9 & 44.8 & 42.5 \\
\hline 60 & 73.8 & 70.4 & 56.4 \\
\hline 80 & 89.1 & 85.6 & 71.1 \\
\hline 100 & 98.7 & 90.1 & 82.8 \\
\hline
\end{tabular}




\section{CONCLUSIONS AND FUTURE WORKS}

This paper focuses on providing adaptive delay tolerant routing protocol for heterogeneous cognitive radio ad hoc networks. Simulations are carried out using cognitive radio cognitive network (CRCN) simulator. The performance metrics such as throughput, packet delivery ratio and delay are taken into account based on pausetime. Simulation results prove that the proposed routing protocol ADTRP has better performance in terms of increased throughput, better packet delivery ratio, decreased packet drop and reduced delay. The protocol can be further extended by incorporating security mechanism in the near future.

\section{REFERENCES}

[1] M. Cesana, F. Cuomo, and E. Ekici, "Routing in cognitive radio networks: Challenges and solutions," Ad Hoc Netw., vol. 9, no. 3, pp. 228-248, May 2011.

[2] G. Cheng, W. Liu, Y. Li, and W. Cheng, "Spectrum aware on-demand routing in cognitive radio networks," in Proc. IEEE DYSPAN, Dublin, Ireland, Apr. 2007, pp. 571-574.

[3] A. Sampath, L. Yang, L. Cao, H. Zheng, and B. Zhao, "High throughput spectrum-aware routing for cognitive radio networks," in Proc. CROWNCOM, Singapore, May 2008, pp. 1-6.

[4] G.-M. Zhu, I. Akyildiz, and G.-S. Kuo, "STOD-RP: A spectrum-tree based on-emand routing protocol for multi-hop cognitive radio networks," in Proc. IEEE GLOBECOM, Nov. 2008, pp. 1-5.

[5] K. Chowdhury and M. Felice, "SEARCH: A routing protocol for mobile cognitive radio ad-hoc networks," Comput. Commun., vol. 32, no. 18, pp. 1983-1997, Dec. 2009.

[6] K. Chowdhury and I. Akyildiz, "CRP: A routing protocol for cognitive radio Ad Hoc networks," IEEE J. Sel. Areas Commun., vol. 29, no. 4, pp. 794-804, Apr. 2011.
[7] A. Cacciapuoti, M. Caleffi, and L. Paura, "Reactive routing for mobile cognitive radio ad hoc networks," Ad Hoc Netw., vol. 10, no. 5, pp. 803-815, Jul. 2012.

[8] B. Karp and H. Kung, "GPSR: Greedy perimeter stateless routing for wireless networks," in Proc. ACM MOBICOM, Boston, MA, USA, Aug. 2000, pp. 243254.

[9] D. Niculescu and B. Nath, "Trajectory based forwarding and its applications," in Proc. ACM MobiCom, San Diego, CA, USA, Sep. 2003, pp. 260-272.

[10] WebReference:http://faculty.uml.edu/Tricia_Chigan/Res earch/CRCN_Simulator.htm.

[11] WebReference:http://www.ece.gatech.edu/research/labs/ bwn/CRAHN/CRAHN_arch.jpg.

[12] X. Jin, R. Zhang, J. Sun, and Y. Zhang, "TIGHT: A Geographic Routing Protocol for Cognitive Radio Mobile Ad Hoc Networks", IEEE Transactions on Wireless Communications, Vol. 13, no. 8, pp. $4670-$ 4681, 2014.

[13] C. Perkins, E. Belding-Royer, and S. Das, Ad Hoc OnDemand Distance Vector (AODV) Routing, RFC 3561, Jul. 2003.

[14] D. Chen and P. Varshney, "A survey of void handling techniques for geographic routing in wireless networks," IEEE Commun. Surveys Tuts., vol. 9, no. 1, pp. 50-67, 2007.

[15] P. Bose, P. Morin, I. Stojmenovic, and J. Urrutia, "Routing with guaranteed delivery in Ad Hoc wireless networks," in Proc. 3rd Int. Workshop DIALM, Aug. 1999, pp. 48-55.

[16] P. Bose, P. Morin, I. Stojmenovic, and J. Urrutic, "Routing with guaranteed delivery in ad hoc wireless networks," Wireless Netw., vol. 7, no. 6, pp. 609-616, Nov. 2001.

[17] D. B. Johnson and D. A. Maltz, "Dynamic source routing in ad hoc wireless networks," Mobile Comput., vol. 353, pp. 153-181, 1996. 